\title{
Review: lack of evidence exists for effectiveness of over the counter cough preparations for children with upper respiratory tract infections
}

\author{
Schroeder K, Fahey T. Should we advise parents to administer over the counter cough medicines for acute cough? \\ Systematic review of randomised controlled trials. Arch Dis Child 2002 Mar;86:170-5. \\ QUESTION: In children with upper respiratory tract infections (URTIs), are over the \\ counter (OTC) cough medicines effective for relieving cough?
}

\section{Data sources}

Studies were identified by searching the Cochrane Acute Respiratory Infections Group specialised register, the Cochrane Controlled Trials Register, Medline (to 1999), EMBASE/Excerpta Medica (to 1999), the U.K. Department of Health National Research Register (December 2000), personal files, and bibliographies of relevant studies; and by contacting authors, pharmaceutical companies, and the Proprietary Association of Great Britain.

\section{Study selection}

2 reviewers independently selected randomised controlled trials (RCTs) if OTC cough preparations were tested in children ( $<16$ y of age) with acute cough $(<3$ wks in duration) associated with URTIs, a placebo group was included, and cough outcomes were reported. Exclusion criteria were chronic cough $(>3$ wks in duration or caused by chronic underlying disease), artificially induced cough in healthy volunteers, or the use of non-conventional or non-oral preparations.

\section{Data extraction}

2 reviewers independently assessed the quality of study methods and extracted data on patient age, sex, and smoking status; cough preparation and dose; definition of illness; and outcomes. Investigators were contacted for additional information when necessary, and studies written in languages other than German and English were translated.

\section{Main results}

6 RCTs (438 children) were included. The quality of study methods was generally low. Studies were not pooled because of the few RCTs in each drug class, the limited quantitative data available, and heterogeneity among RCTs. Antitussives: 1 RCT $(n=57)$ showed that a single dose of dextromethorphan (cough score reduction 2.1) or codeine (cough score reduction 2.2) for 3 nights was not more effective than placebo (cough score reduction 2.2). Mucolytics: 1 RCT $(\mathrm{n}=40)$ showed that letosteine improved symptom scores better than placebo from day 4 to day 10 (mean difference 0.2 points, $P<0.01)$. Antihistamine-decongestant combinations: 1 RCT $(n=59)$ showed that brompheniramine plus phenylpropanolamine did not reduce the number of children coughing 2 hours after each dose better than placebo
$(49 \%$ v $43 \%, P=0.66)$. A second RCT $(\mathrm{n}=96)$ showed that brompheniramine plus phenylephrine plus phenylpropanolamine did not improve cough symptoms better than placebo $(67 \% v 58 \%, p>0.2)$ or no treatment $(67 \%$ v $70 \%, p>0.2)$. Other drug combinations: 1 RCT tested 2 pediatric cough syrups (Triaminicol syrup and Dorcol pediatric cough syrup) in 43 children. Satisfactory response reported by parents did not differ between treatment and placebo $(69 \%$ for both treatment groups $v 57 \%$ for placebo, $P>0.2)$. Antihistamines: In 143 children, clemastine and chlorpheniramine did not differ from placebo for improvement in physician-rated cough scores (40\% for both treatment groups $v 28 \%$ for placebo, $p=0.2)$.

\section{Conclusion}

In children with acute cough associated with upper respiratory tract infection, the existing evidence does not support the effectiveness of over the counter cough preparations.

\section{COMMENTARY}

Acute cough in children is common, and the implications of use or non-use of cough medicines are important in terms of both patient care and expense. The paucity and poor quality of the studies in the review by Schroeder and Fahey is a sad reflection of research priorities. The results are remarkably consistent, and the stronger conclusion that cough medicines are not effective might be drawn were the trials not so heterogeneous. However, it may be unwise to generalise too much across a wide age spectrum of children and a variety of drugs. Dosage of medication in relation to the age and size of the child and the duration of cough in days at administration onset are vital factors that are not considered. More and better evidence is needed.

In particular, future research should address 2 issues: firstly, are there risks (other than adverse drug effects) in suppression of the defensive cough reflex during acute URTI? Secondly, are the new and novel antitussive compounds that are being developed effective and safe in humans? ${ }^{1}$

Charles Bridges-Webb, MBBS MD University of Sydney Sydney, New South Wales, Australia Chung KF, Chang AB. Therapy for cough: active agents.
Pulm Pharmacol Ther. 2002;15:335-8.
S Primary Care Career Scheme.

For correspondence: D K Schroeder, University of Bristol, Bristol, UK. Email k.schroeder@ bristol.ac.uk. 